\title{
Maps with Least Distortion Between Surfaces: From Geography to Brain Warping
}

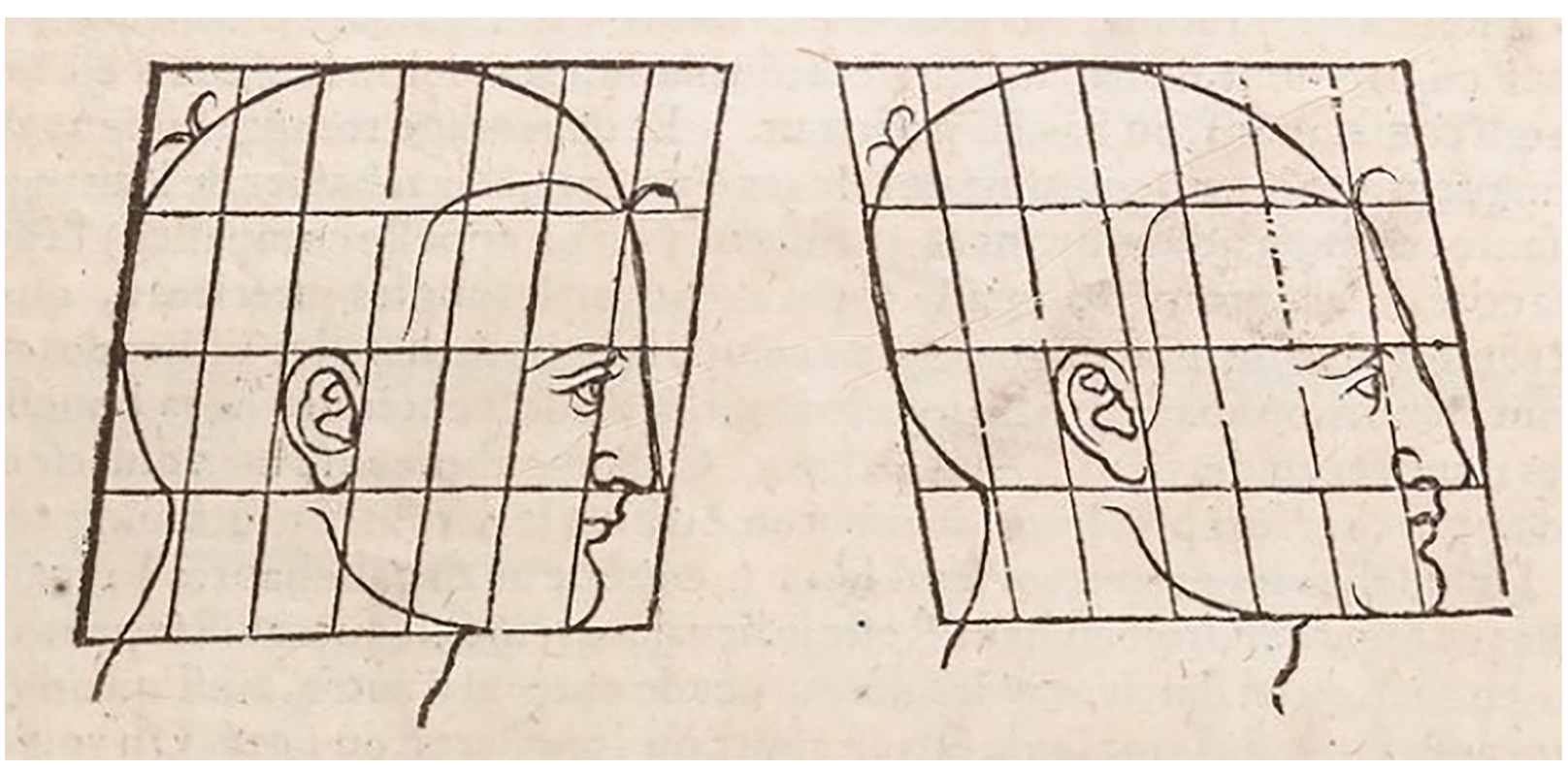

\section{Athanase Papadopoulos}

\section{Introduction}

Many geometrical problems find their origin in practical ones. Darboux writes in his 1908 ICM talk titled "Les origines, les méthodes et les problèmes de la géométrie infinitésimale" (The origins, methods and problems of infinitesimal geometry) [4]: "Like many other branches of human knowledge, infinitesimal geometry was born in the study of practical problems." He goes on explaining how problems of cartography, that is, the art of representing regions of the surface of the earth on a Euclidean piece of paper, led to important developments in geometry made by Lagrange, Euler, Lambert, Gauss, Beltrami, Chebyshev, and others. In fact, Darboux himself was most interested in cartography. In a memorial article read at the French

Athanase Papadopoulos is Directeur de Recherche, CNRS, Strasbourg, France, and Lamé Chair Professor, Chebyshev Laboratory, St. Petersburg State University, Russia. His email address is athanase.papadopou1os@gmai 1 . com.

For permission to reprint this article, please contact:

reprint-permission@ams.org

DOI: https://doi.org/10.1090/noti1979
Academy of Sciences on December 10, 1917, Picard recalls that Darboux had planned to write a book on this subject, which, he says, seduced him because of its elegance and practical importance. ${ }^{1}$ It is no accident that the vocabulary concerning manifolds and geometric structures includes terms such as "map," "chart," and "atlas."

The mathematical problem of cartography is that of finding maps that optimize distortion in a sense that needs to be made precise. Indeed, the word "distortion" is attached to a variety of parameters-distances, areas, and angles - and the general problem is to find mappings that are closest to mappings that make a compromise between these parameters among all mappings from a given subset of the sphere onto the Euclidean plane. Furthermore, geographers usually had additional constraints on the desired maps, such as preserving distances along a certain meridian or along all meridians, or of sending parallels (circles centered at the poles) to parallel straight lines or to concentric circles or to other types of "parallel" curves in

${ }^{1}$ The address is published in a compendium of short texts by Picard: Émile Picard, Discours et Mélanges, Paris, Gauthier-Villars, 1922. 
the plane. We shall mention examples of mappings from the sphere to the Euclidean plane satisfying such requirements.

The various approaches to the question of maps with minimal distortion from (subsets of) the sphere onto the Euclidean plane acted as a motivation for Euler, Lagrange, Gauss, Chebyshev, and other geometers to study general maps between differentiable surfaces. Furthermore, geography gave rise to an early version of extremal quasiconformal mappings as a particular class of least-distortion mappings. This took place in the nineteenth century, before quasiconformal mappings were officially introduced in the late 1920s as a tool in conformal geometry and before they led to the first known metric on Teichmüller space. Thurston's theory of best Lipschitz maps, developed in his paper "Minimal stretch maps between hyperbolic surfaces" [19], is also based on the idea of studying maps with least distortion between surfaces. It led him to the definition of another metric on Teichmüller space, the Thurston metric, which is today an active research topic. We shall discuss all this in the present paper. We shall also see how least distortion maps occur in art, biology, and the medical sciences, and in particular in brain imaging.

A common feature of all the maps we mentioned is the use of transformations of underlying systems of coordinates that make these maps geometrically defined in a simple way; this is the theme we want to emphasize here. At the same time, we shall illustrate Darboux's claim that several geometrical problems were motivated by practical ones.

\section{Old Cartography}

In mathematical terms, the object of cartography is to draw mappings with least distortion from regions of the sphere to the Euclidean plane. This art and science was developed in Ancient Greece by mathematicians and geographers, and it arose from practical needs. Indeed, maps of the earth and of the celestial sphere were useful for navigation, land surveying, the knowledge of the exact time of the day, computation of dates of eclipses, etc. The fact that the relative magnitudes of lands needed to be represented in a close-to-faithful manner came naturally from needs linked to harvesting, the distribution of water, tax calculation, etc. The question of finding the "best" maps, that is, those with minimal distortion, arose very naturally.

There was speculation about whether the world was finite or infinite, but this did not affect the cartography of the celestial dome, which was identified with a sphere whose radius is irrelevant (and might be infinite): the distance between two points on the sphere is the angle made by two rays starting at the observer's eye and joining them. It was known that the diameter of the earth was small enough compared to its distances to the celestial bodies so

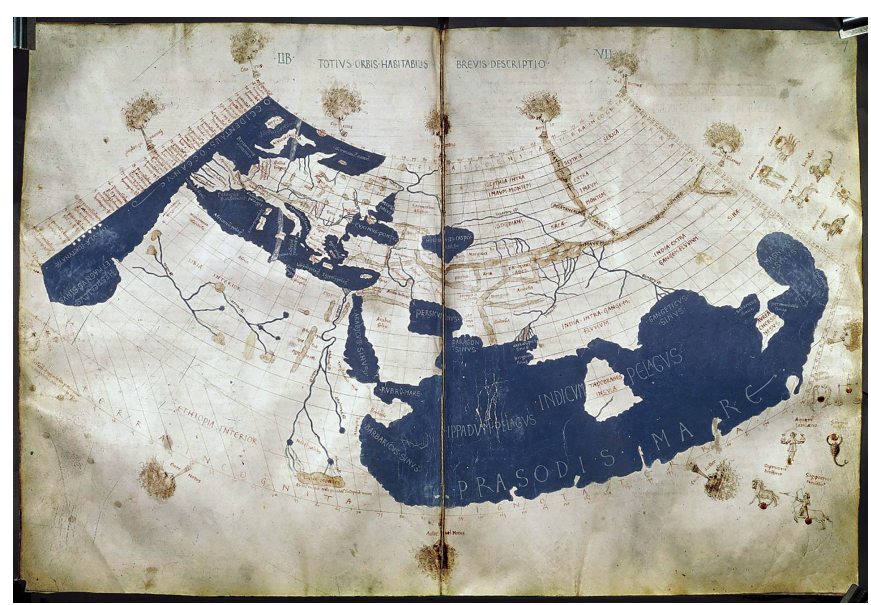

Figure 1. A world map from a fifteenth-century manuscript; drawing attributed to Francesco di Antonio del Chierico, British Library.

that the choice of the point at which the rays start makes no significant difference.

Several preeminent mathematicians of Greek antiquity were at the same time geographers and astronomers. From the known results in spherical geometry, it was easy for them to conclude that, except in very special cases, it is not possible to represent a subset of the sphere onto the plane by a map that preserves distances up to scaling. The notion of conformal (that is, angle-preserving) mapping was well known. The stereographic projection with center a point on the sphere onto a plane containing the great circle having that point as pole was probably the most popular projection from the sphere onto a Euclidean plane. ${ }^{2}$ It was already used by Hipparchus back in the second century BC. ${ }^{3}$ This projection is not the most useful one, because it distorts significantly distances and areas at points that are close to the projection center.

Among the mathematicians of Greek antiquity who were at the same time geographers, the name of Claudius Ptolemy (second century AD) comes to the forefront. Besides his major mathematical work called the Mathematical Syntaxis (later known as the Almagest), his Geography is a treatise that had an enormous influence. Ptolemy developed there the theory of geographical maps using mathematical tools of his predecessors and astronomical tables he compiled. His Planisphaerium, which survives in Arabic translations, may be regarded as a treatise on the stereographic projection.

Ptolemy's Geography reached us through medieval edi-

\footnotetext{
${ }^{2}$ Sometimes, cartographers used a stereographic projection onto a plane tangent to the sphere at the point diametrically opposite to the center of the projection.

${ }^{3}$ For the use of the stereographic projection in Greek antiquity, the reader may refer to Delambre's Histoire de l'astronomie ancienne, Courcier, Paris, 1817 (Vol. 1, p. 184ff), or d'Avezac's "Coup d'œil historique sur la projection des cartes de géographie (suite et fin)," Bull. Soc. Géographie, 5 e série, t. V (1863), pp. 437-485.
} 


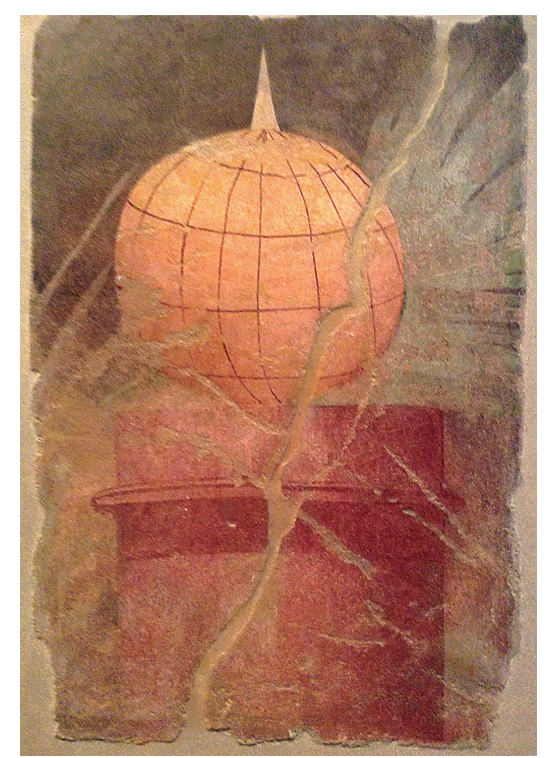

Figure 2. Celestial globe with two perpendicular foliations, Roman, c. 50-40 BC, Metropolitan Museum of Art, New York.

tions. Figure 1 is a map drawn of the oikouménê (the known world) in the second century $\mathrm{AD}$, reconstructed from Ptolemy's Geography in fifteenth-century Florence, based on earlier manuscripts. In this projection, the small circles on the sphere that are parallel to the equator (the parallels) are sent to ellipses, whereas the great circles passing through the North and the South poles (the meridians) are sent to Euclidean lines.

Figure 2 is a representation of a celestial globe from the first century BC reduced to its most basic element: the two perpendicular foliations of the coordinate system. The parallels and the meridians of the sphere form a pair of transverse foliations (with singular points at the poles), and drawing the images of these two foliations is a way of describing the map.

During the Renaissance, the need for geographical maps was intensified by the discovery of new lands. Leonardo da Vinci (1452-1519) and Albrecht Dürer (1471-1528), besides being two of the greatest artists of their times, were remarkable mathematicians who were highly interested in geography. They worked on the technical problems raised by map drawing. They also drew geographical maps whose esthetic value is highly praised. We shall talk more about Dürer later in this article.

Figure 3 is a picture of the heart-shaped map "Recens et integra orbis descriptio" (Recent and complete description of the world) by the French mathematician and astronomer Oronce Fine. ${ }^{4}$ He drew it between 1534 and 1536. By this projection, the parallels are sent to a foliation of the heart whose leaves are close to circles near

${ }^{4}$ Oronce Fine (1494-1555), or Oronteus Finaeus, was the first to hold the chair of mathematics at the Collège de France.

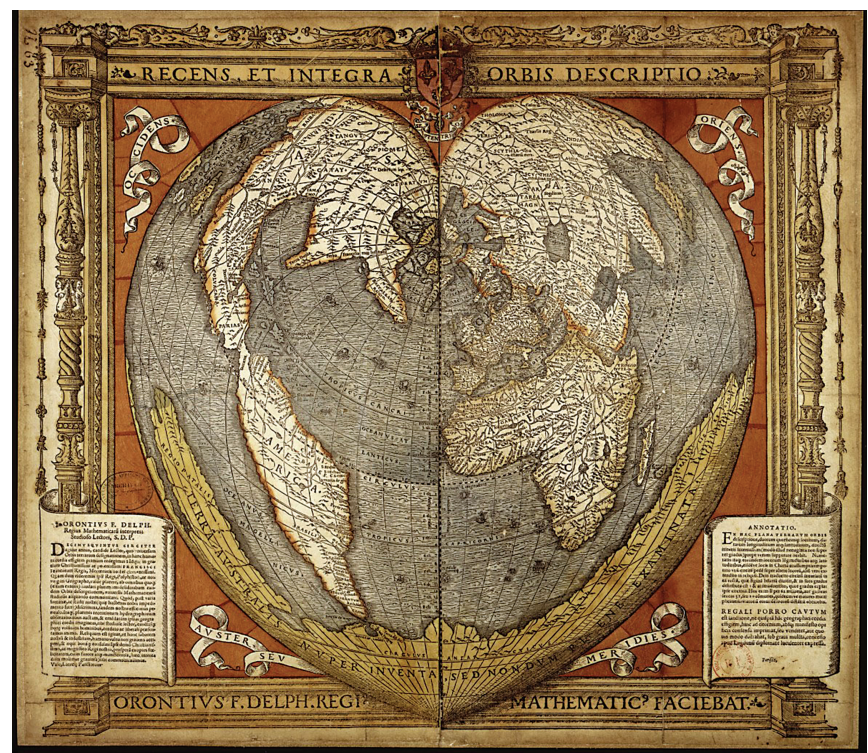

Figure 3. A world map, drawn between 1534 and 1536 by Oronce Fine, Bibliothèque Nationale de France.

the North Pole and to straight lines near the South Pole. The meridians are sent to lines that are everywhere almost perpendicular to the images of the parallels. One property of this map is that the distances along the parallels and along the central meridian are preserved up to scaling. Such a projection is known as a Stabius-Werner projection, in honor of the cartographers Johannes Stabius (1460-1522), who was the first to highlight it, and Johannes Werner (1466-1528), who wrote a treatise on it. The shape of this geographical map has several practical advantages, one of them being that it is agnostic on the question-whose answer was unknown at that timeof whether North America and Asia are connected or not. This map is inspired by a drawing of Ptolemy, and its conception uses advanced spherical geometry and geometrical constructions combining the stereographic and the gnomonic projections; see the discussion in [10].

\section{Modern Cartography}

The invention of analytic geometry by Descartes and Fermat and of differential calculus by Newton and Leibniz completely transformed the bases of mathematical cartography. The definitions of a scaling factor, of deviation from conformality, and of other distortion properties of a map were gradually introduced, based on the concept of derivative. Differential equations were used to study the existence of geographical maps whose pointwise distortion satisfies specific properties. Cartography became gradually part of the theory of differential geometry of surfaces.

Euler, besides being the most preeminent mathematician at the Academy of Sciences of St. Petersburg, was thoroughly engaged in cartography. For several years, he as- 
sisted the famous French geographer and astronomer Joseph-Nicolas Delisle (1688-1768) in the huge project of drawing geographical maps of the new Russian Empire. In 1777, he published three memoirs that established the foundations of the modern field of cartography. He addressed there the basic questions in terms of differential calculus and the calculus of variations. In one of these memoirs, Euler emphasizes the fact that he considers arbitrary maps from the sphere to the plane: "Any point of the surface of the sphere is represented on the plane by any desired rule" $[5, \S 1]$. This is probably one of the first explicit occurrences of mappings that are not necessarily given by analytic formulae. Euler then considers the question of the nonexistence of a "perfect" map. ${ }^{5}$ His approach to the problem consists of translating the given geometrical conditions into a system of differential equations and showing that this system does not have a solution. After he shows the nonexistence of a perfect map, Euler examines in detail several projections from the sphere to the Euclidean plane, searching systematically for the partial differential equations they satisfy. The classes of maps he considers include conformal mappings (Euler calls them "similitudes on the small scale"), area-preserving mappings, and mappings where the images of all the meridians are perpendicular to a given axis while those of all parallels are parallel to it. In each case, he gives examples and he studies their distance and angle distortion.

In the memoir [6], Euler explains the advantage of a projection discovered by Delisle, and he develops the mathematical theory behind it. He formulates the problem under consideration as a problem of "minimizing the maximal error" over an entire region. He shows that in Delisle's projection, while the meridians are represented by straight lines, the images of the other great circles "do not deviate considerably from straight lines" [6, $\$ 22]$. This is probably the first time where we encounter the notion of a map sending geodesics to "quasigeodesics" (without the name).

\footnotetext{
${ }^{5}$ The question of what a "perfect map" is in the sense of Euler is a subject of confusion. Wrong statements are attributed to him in the literature. R. Ossermann, in his paper "Mathematical mapping from Mercator to the millennium" (Spectrum, MAA, 2004) attributes the following theorem to Euler: It is impossible to make an exact scale map of any part of a spherical surface. By an exact scale map, Osserman means a map that preserves distances up to scale, and he refers to Euler's paper [5]. However, the result that Osserman states was already known to the Greeks from elementary spherical geometry, and Euler certainly knew that. In fact, by a perfect map, Euler meant a smooth map from a sphere to the Euclidean plane such that any point in the domain has a neighborhood on which the restriction of the map preserves distances infinitesimally along the meridians and parallels and preserves angles between these lines. The same confusion about Euler's result occurs in the paper "Curvature and the notion of space" by A. Knoebel, J. Lodder, R. Laubenbacher, and D. Pengelley (in Mathematical Masterpieces, Springer Verlag, 2007, pp. 159-227) and in several other papers written by historians of mathematics. Euler's theorem is carefully analyzed and presented in modern terms in the recent paper [2].
}

Such a property reappeared almost a century later in a paper by Beltrami [1]. ${ }^{6}$

Lagrange published two memoirs on cartography [11] in which he refined Euler's research and that of Lambert. As a general rule, Lagrange replaced Euler's analytic arguments by geometric ones.

In the first memoir, Lagrange surveys various projections of the earth and of the celestial sphere that were in use at his time. The variety of geographical maps leads him to the general question, What is a general mapping between two surfaces? This is a two-dimensional analogue of the question What is a function? which was the subject of a debate that lasted several decades and which involved the best mathematicians of the eighteenth and nineteenth centuries: Euler, Lagrange, d'Alembert, Riemann, Fourier, etc.

Lagrange mentions the works of Ptolemy and Euler, but especially those of Lambert, who, according to him, was the first to study arbitrary angle-preserving maps from the sphere to the plane and who, in his memoir [12], gave a characterization of least-distortion maps among those that are angle-preserving. Lagrange then studies geographical maps that are not necessarily angle-preserving and where the images of the meridians and the parallels are not required to be circles or lines. They can be, using his terms, arbitrary "mechanical lines." This means that they are not necessarily given by a formula. He declares that a geographical map is determined once these images-our familiar pair of transverse foliations-have been determined. This is an obvious fact, but the idea is highly appealing. It makes the relation with our exposition in the section "Art and Biology," where maps are described in terms of their action on a grid of perpendicular lines.

In the same paper, Lagrange gives a formula for the local distortion factor of a conformal map. This is a function on the sphere that measures the length distortion at each point. It is defined at each point as the ratio of the infinitesimal length element at the image by the infinitesimal length element at the source. The fact that the map is conformal makes this quantity well defined. ${ }^{7}$ Lagrange then gives a formula for the distortion of maps that send meridians and parallels to circles or to circles and straight lines.

Lagrange's formulae were used by Pafnouti Chebyshev (1821-1894), who was a devoted reader of Euler, Lagrange, Gauss, Abel, and other mathematicians that preceded him. We learn from Chebyshev's biographer that he avoided reading the mathematical works of his contemporaries, considering that this would prevent him from having origi-

\footnotetext{
${ }^{6}$ Motivated by geography, Beltrami also considered the problem of mapping a given surface onto the plane in such a way that the geodesics of the surface are sent to straight lines, and he showed that this is not possible unless the surface has constant curvature.

${ }^{7}$ The formula for the local distortion is given on p. 646 of Lagrange's paper [11].
} 
nal ideas. ${ }^{8}$ Like Euler, Chebyshev was interested in almost all branches of pure and applied mathematics. His Collected Papers contains two papers on cartography [3], both called "Sur la construction des cartes géographiques." It is interesting to read some sentences from the beginning of the second paper; they remind us of the passage from Darboux's 1908 ICM lecture we quoted at the beginning of the present article. Chebyshev writes: "Today, [mathematical sciences] produce a greater interest because of their influence on art and industry. Not only practice makes profit of these relations: conversely, science itself grows under the influence of practice." He then mentions the importance of the problem of constructing geographical maps, making relations with the problem of heat distribution and other problems.

In the first paper, Chebyshev, using Lagrange's formula for the local distortion of a conformal map, addresses the problem of finding conformal geographical maps whose local distortion is minimal. He defines a "magnification ratio" ("rapport d'agrandissement") at each point, based on Lagrange's notion of local distortion. He makes the relation with the Laplace equation and reduces the problem of finding geographical maps that have least distortion to a problem in potential theory. His main result, stated in modern terms, is that any simply connected open subset of the sphere bounded by a twice differentiable curve can be conformally mapped to the Euclidean plane by a map that is distortion minimizing and that such a map is unique up to similarity. Furthermore, this map is characterized by the fact that its magnification ratio is constant on the boundary curve of the domain [3, p. 235]. In the introduction to his second paper, Chebyshev places the problem of cartography in the context of one of his favorite subjects: optimization theory.

Milnor, in a paper titled "A problem in cartography," gave another proof of Chebyshev's theorem. After reviewing this result, he writes: "This result has been available for more than a hundred years, but to my knowledge it has never been used by actual map makers." We shall say more about Milnor's work below.

\section{Art and Biology}

We consider now other kinds of maps between surfaces, and for this we return to Renaissance art, more especially, to Albrecht Dürer, whose drawings will lead us directly to D'Arcy Thompson's diagrams for growth and form in biology.

Closely related to the subject of our article are Dürer's drawings represented in Figures 4 and 5. They are extracted from his treatise titled Four Books of Human Proportions

${ }^{8}$ Cf. C. A. Possé, "Excerpts of a biography of Chebyshev," contained in his Collected Works, edited by A. Markoff and N. Sonin, Vol. 2, pp. I-VI.

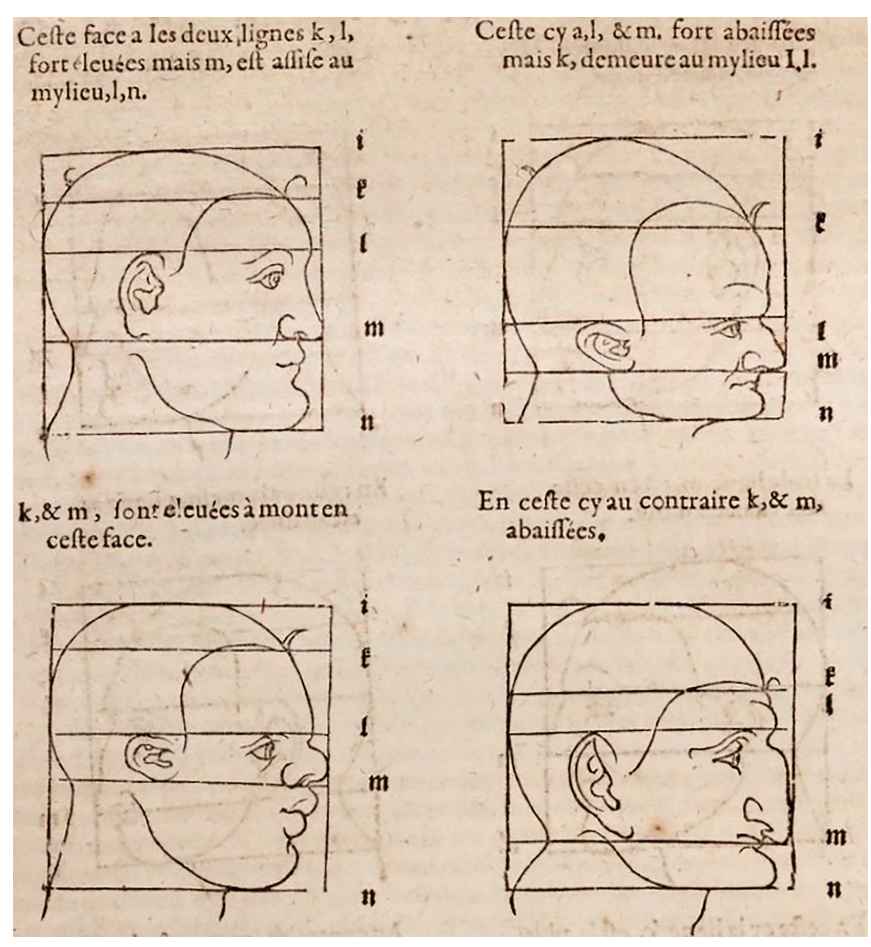

Figure 4. From Dürer's Four Books of Human Proportions (Nuremberg, 1528).

(Nuremberg, 1528). ${ }^{9}$ The pictures are self-explanatory: the head is divided into a certain number of significant parts (the top of the head, the forehead, the part containing the eyes and the nose, the part containing the mouth and the chin), and each 2-dimensional transformation is divided into transformations between these parts. ${ }^{10}$ At first approximation, these transformations are affine. ${ }^{11}$

In the diagram represented in Figure 5, the heads are transformed using grids that are not rectangular.

An important part of Dürer's treatise is dedicated to drawings of the human body based as well on proportions and transformations between parts of this body. Like the head, the body is divided by lines that are meaningful: starting from the top, the division lines pass through the top of the head, the forehead, the eyebrows, the nose, the chin, the top of the shoulders, etc., until the ankle and the sole. Goethe, as a biologist and observer of nature, highly prized Dürer's proportional transformations. ${ }^{12}$

From Dürer's grid transformations, which allow a mean-

\footnotetext{
${ }^{9}$ We are using the French version, Les quatre livres d'Albert Durer, Iean Ieantz, Arnheim, 1614.

${ }^{10}$ Dürer was certainly thinking of 3-dimensional transformations, but this does not change our discussion much.

${ }^{11}$ In fact, the maps between the pieces are not affine: if they were so, then the resulting contour lines would not be smooth. Dürer gives a precise geometric construction for the maps between the various pieces in Book III of the treatise (p. 77ff).

${ }^{12}$ Goethe mentions Dürer in his 1776 poem "Hans Sachsens poetische Sendung."
} 


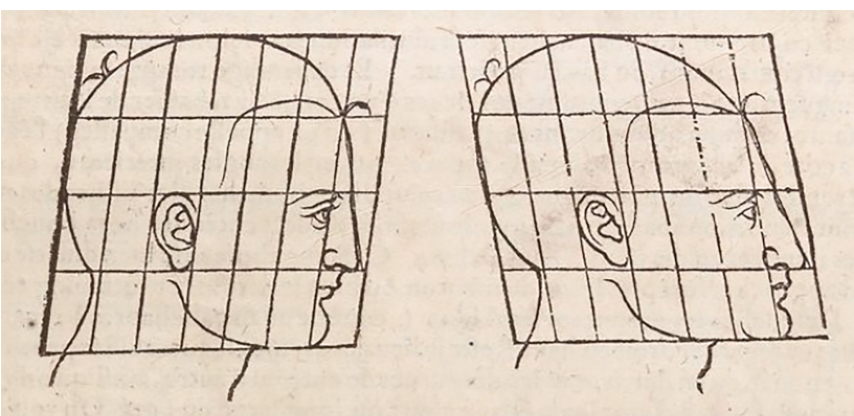

Figure 5. From Dürer's Four Books of Human Proportions (Nuremberg, 1528)

ingful comparison between forms, we pass to those of the Scottish biologist D'Arcy Thompson.

In 1917, D'Arcy Wentworth Thompson (1860-1948) published the first edition of a groundbreaking book, On Growth and Form [16], in which he developed a theory of change of form in biology based on simple geometric transformations that use, like Dürer's drawings, deformation of a grid formed by two transverse families of lines. Thompson declares on several occasions that he was inspired by the drawings of fifteenth-century artists such as LeonBattista Alberti, Albrecht Dürer, and Leonardo da Vinci. On p. 80 of his treatise, he writes:

[The general connection between growth and form] is implicit in the "proportional diagrams" by which Dürer and his brother-artists illustrated the change in form, or of relative dimensions, which mark the child's growth to boyhood and to manhood.

Chapter XVII, which is the last chapter of D'Arcy Thompson's book, is titled "On the Theory of Transformations, or the Comparison of Related Forms." It contains an exposition of the author's most interesting ideas on morphology, a science whose name he borrowed from Goethe and whose methods and principles he considered to be derived from mathematics. He writes (p. 1027): "We are apt to think of mathematical definitions as too strict and rigid for common sense, but their rigour is combined with all but endless freedom." In the long introduction to his chapter, he comments on the relation between mathematics and experimentation, quoting Gauss, who called mathematics "a science of the eye"; Sylvester, who said that "most, if not all, of the great ideas of modern mathematics have had their origin in observation"; and several other mathematicians. D'Arcy Thompson also declares that Descartes invented his method of coordinates as a generalization of the Renaissance "proportional diagrams." About form, he says:

We never even seek for a formula to define this fish or that, or this or that vertebrate skull. But we may already use mathematical language to de- (a)
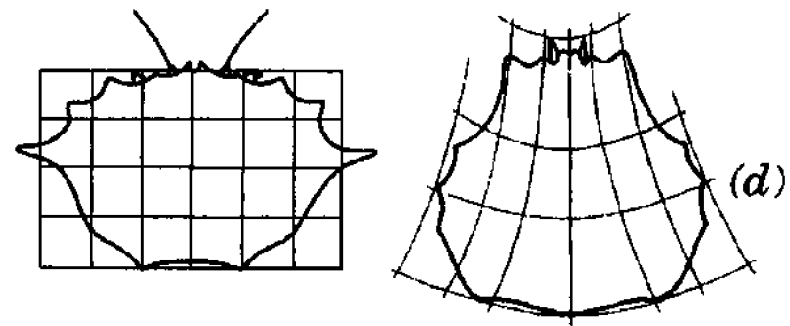

(b)
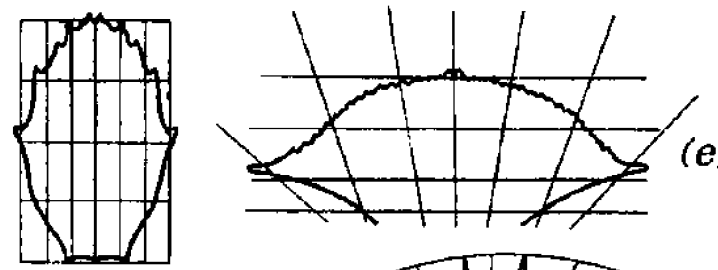

(c)

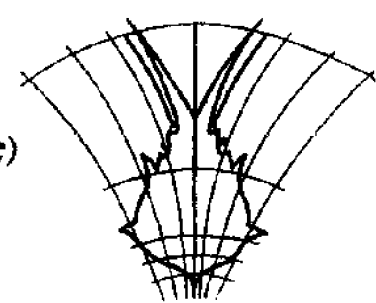

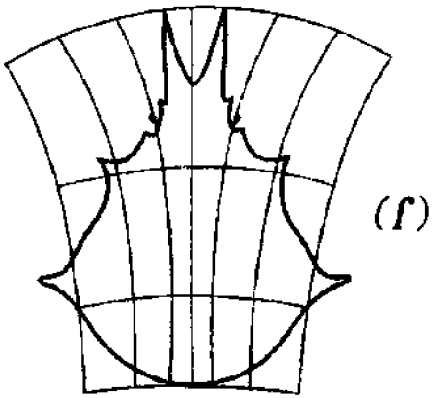

(r)
Figure 6. Maps between shells, from D'Arcy Thompson's Growth and Form.

scribe, even to define in general terms, the shape of a snail-shell, the twist of a horn, the outline of a leaf, the texture of a bone, the fabric of a skeleton, the stream-lines of fish or bird, the fairy lace-work of an insects' wing.... In a very large part of morphology, our essential task lies in the comparison of related forms rather than in the precise definition of each; and the deformation of a complicated figure may be a phenomenon easy of comprehension, though the figure itself has to be left unanalysed and undefined.

[This process of comparing forms] lies within the immediate province of mathematics, and finds its solution in the elementary use of a certain method of the mathematician.

D'Arcy Thompson, in his development, uses conformal transformations and discusses isothermal coordinates (see in particular p. 1047 of [16]). His theory is based on geometric transformations that are exemplified in Figures 6 and 7, which we have reproduced from his book. He writes:

The problem is closely akin to that of the cartographer who transfers identical data to one projection or another, and whose object is to secure (if it be possible) a complete correspondence, in each small unit of area, between the one representation and the other. The morphologist will not seek to 

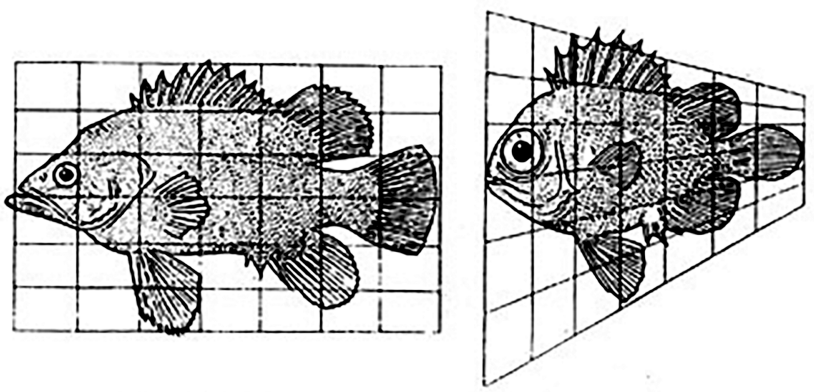

Fig. 150. Polyprion.

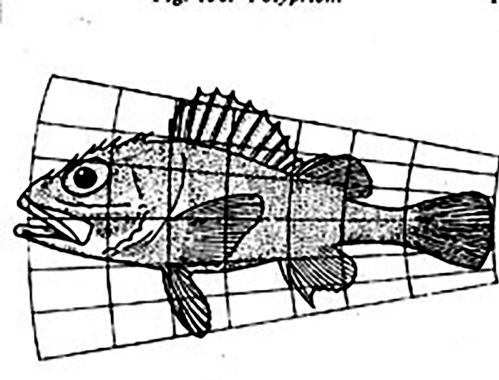

Fig. 152. Scorpaena sp.
Fig. 151. Pseudopriacanthus altus.

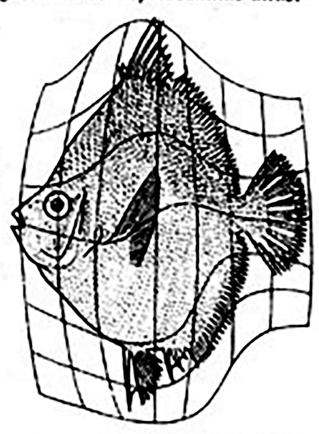

Fig. 153. Antigonia capros.

Figure 7. Maps between fish, from D'Arcy Thompson's Growth and Form.

draw his organic forms in a new and artificial projection; but, in the converse aspect of the problem, he will enquire whether two different but more or less obviously related forms can be so analysed and interpreted that each may be shown to be a transformed representation of the other.

On several occasions, René Thom expressed his admiration of D'Arcy Thompson. He referred to diagrams such as those represented in Figure 7 as examples of mappings between stratified spaces. ${ }^{13}$ Thom's paper "L'explication des formes spatiales : réductionnisme ou platonisme? La morphogenèse" (Paris, Malouine-Doin, 1980) concerns the general notion of form and its classification. For him, one of the fundamental problems in morphology is to make precise, from the mathematical point of view, the equivalence relation saying that two closed subsets of a Euclidean four-dimensional space (parametrizing space and time) have the same form. The relation satisfies certain metrical constraints that he says "are generally impossible to formalize," and he refers to the "congruences in the sense of D'Arcy Thompson."

Claude Levi-Strauss, the founder of structuralism in anthropology, ethnology, and linguistics, an approach to these fields based on the idea that there is a universal system of deep structures that underlies them and that transcends the separation between cultures, acknowledged on

\footnotetext{
${ }^{13}$ See for instance Chapter 5, $\$$, of Thom's Esquisse d'une sémiophysique and his 1988 Solignac lecture notes (Éditions du CNRS, 1988).
}

several occasions his debt to D'Arcy Thompson. In a book of interviews with him, ${ }^{14}$ his interlocutor recalls that the notion of "transformation" occupies a crucial role in his essay "La pensée sauvage" and his four-volume work Mythologiques, which is considered as the absolute reference in the field. He asks him, "From whom did you borrow this notion? From logicians?" Levi-Strauss's answer is, "Neither from logicians nor from linguists. It came to me from a book which played a decisive role for me, On Growth and Form.... It was an illumination." In the same interview, Levi-Strauss mentions Dürer, Goethe, together with D'Arcy Thompson (p. 160).

\section{Extremal Quasiconformal Mappings and Minimal Stretch Maps}

Geography led to the first rudiments of quasiconformal mappings, that is, mappings with bounded conformal distortion. This notion (without the name) occurs in the work of the nineteenth-century French mathematician and cartographer Nicolas-Auguste Tissot (1824-1897), whose name is poorly known among mathematicians but well known to geographers.

Tissot introduced a graphical device known under the name Tissot indicatrix. This is a representation of a field of ellipses on a geographical map where, at each point, the ellipse (assumed to be infinitesimal) is the image of an infinitesimal circle in the domain. ${ }^{15}$ The ellipses are characterized by two parameters: their relative size and the ratio of their two axes (the major axis divided by the minor axis). These parameters represent respectively the local area distortion and angle distortion of the map. Thus, the Tissot indicatrix contains information on the distortion of the projection at the points where it is drawn, both in direction and in magnitude.

Tissot studied extensively, from the differentialgeometric point of view, distortions of mappings from the sphere onto the Euclidean plane, but he also developed a theory for the distortion of mappings between general surfaces. His work on the Tissot indicatrix makes him a direct precursor of the theory of quasiconformal mappings. Herbert Grötzsch (1902-1993), who is usually considered to be the founder of the modern theory of quasiconformal mappings, and Oswald Teichmüller (1913-1943), who developed this field extensively, both quote Tissot. In particular, Grötzsch mentions Tissot several times in his 1930 paper [9], in which he studies extremal quasiconformal representations of multiconnected domains, that is, map-

\footnotetext{
${ }^{14}$ De près et de loin (interviews with with Didier Eribon), Odile Jacob, 1988, p. 158.

${ }^{15}$ The expression "infinitesimal circle" means a circle on the tangent space at a point. The map is assumed to be differentiable so that it acts on tangent spaces, sending infinitesimal circles centered at the origin to infinitesimal ellipses. The form and the inclination of the image ellipse do not depend on the radius of the circle.
} 
pings that minimize the maximal conformal distortion. In the figures of that paper, the Tissot indicatrix is represented. The relative size and the directions of the major and minor axes of this ellipse constitute important elements in some of his results. We have reproduced one of his figures in Figure 8 here: On the left-hand side, we have a triply connected region in the complex plane, namely, the unit disc with a shaded region and an arc deleted. On the righthand side, we have another triply connected region in the complex plane: the unit disc with a smaller disc centered at the origin and a circular arc centered at the same point deleted. Grötzsch describes an extremal quasiconformal map between the two regions. On the figure to the right, the Tissot indicatrix is described by its great and small axes.
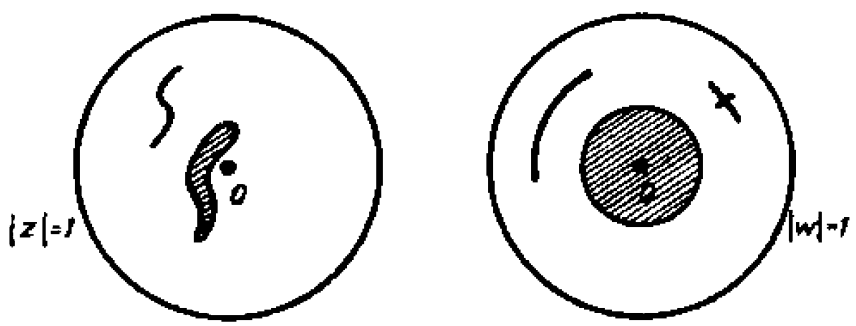

Figure 8. A figure from Grötzsch's paper [9] where the Tissot indicatrix is drawn. The direction of its minor axis points toward the origin.

Teichmüller, in his fundamental paper "Extremale quasikonforme Abbildungen und quadratische Differentiale" (1939), has a section on the origin of quasiconformal mappings in which he declares that the theory started with cartographers, and he mentions Tissot. ${ }^{16}$

Unlike Lagrange, Euler, and Chebyshev, who essentially studied the metric distortion of conformal maps between surfaces, Tissot studied extensively distortion-minimizing nonconformal maps. He proved in particular that for any mapping between two surfaces, there exists, at each point of the domain, a pair of orthogonal directions that are sent to a pair of orthogonal directions, and that unless the mapping is angle-preserving, these orthogonal directions are unique. Assuming the map is not angle-preserving at any point, the families of orthogonal directions on the two surfaces give a pair of unique orthogonal foliations on each surface that are preserved by the mapping. Thus, we are again in the realm of transformations that can be visualized using two transverse foliations, as in geography, in Dürer's drawings, and in those of D'Arcy Thompson.

After extremal quasiconformal mappings, I would like to talk about best Lipschitz maps between surfaces. I will start with some work of Milnor, which was also motivated by cartography.

\footnotetext{
${ }^{16}$ Section 164, p. 479 of the English translation, which appeared in the Handbook of Teichmüller Theory, Vol. V, Zürich, 2015.
}

In his paper [14], Milnor gives the following version of the distortion of maps between surfaces: Consider a map $f: U \rightarrow E$ where $U$ and $E$ are metric spaces with distance functions $d_{U}$ and $d_{E}$. The scale of $f$ with respect to two arbitrary distinct points $x$ and $y$ in $U$ is defined as

$$
\frac{d_{E}(f(x), f(y))}{d_{U}(x, y)} \text {. }
$$

The minimum scale of $f$ is the quantity

$$
\sigma_{1}=\inf _{x \neq y} \frac{d_{E}(f(x), f(y))}{d_{U}(x, y)},
$$

and its maximum scale is the quantity

$$
\sigma_{2}=\sup _{x \neq y} \frac{d_{E}(f(x), f(y))}{d_{U}(x, y)} .
$$

The two quantities $\sigma_{1}$ and $\sigma_{2}$ may be zero, positive, or infinite.

Milnor then defines the distortion of $f$ as

$$
\delta=\log \frac{\sigma_{2}}{\sigma_{1}} .
$$

If the distortion is zero, then the map has constant scale.

Motivated by the problem of drawing geographical maps, Milnor addresses the question of studying maps whose distortion is minimal among all maps from a given subset of the sphere to the Euclidean plane. He proves existence using elementary topology and he addresses the question of uniqueness of such a map, of its differentiability (assuming the domain of the map is such that differentiability makes sense), of its constructiveness, and of how to estimate the distortion of a minimal-distortion map associated to a given domain of the sphere. He obtains results in the case where the subset of the sphere is a disc. He mentions that in this case, the map was known to geographers as the azimuthal equidistant projection: it preserves distances and directions from the center of the disc. In the general case, the questions are open.

Milnor's definition can be adapted to define distance functions on various spaces of metric spaces (for instance, simply connected open subsets of the Euclidean plane). One can then study the properties of such distance functions: existence of geodesics between two arbitrary points in such a space of metric spaces, large-scale geometry, etc. Experience shows that it is not easy to compute Lipschitz distortions and minimal distortions for maps between surfaces, even in the simple cases.

In his paper "Minimal stretch maps between hyperbolic surfaces" [19], Thurston developed a theory of best Lipschitz maps between hyperbolic surfaces and used them to define a new metric on Teichmüller space, which now bears the name Thurston's metric. The theory is parallel to that of the Teichmüller metric in which distances are defined using the quasiconformal instead of the Lipschitz dilatation of mappings. 


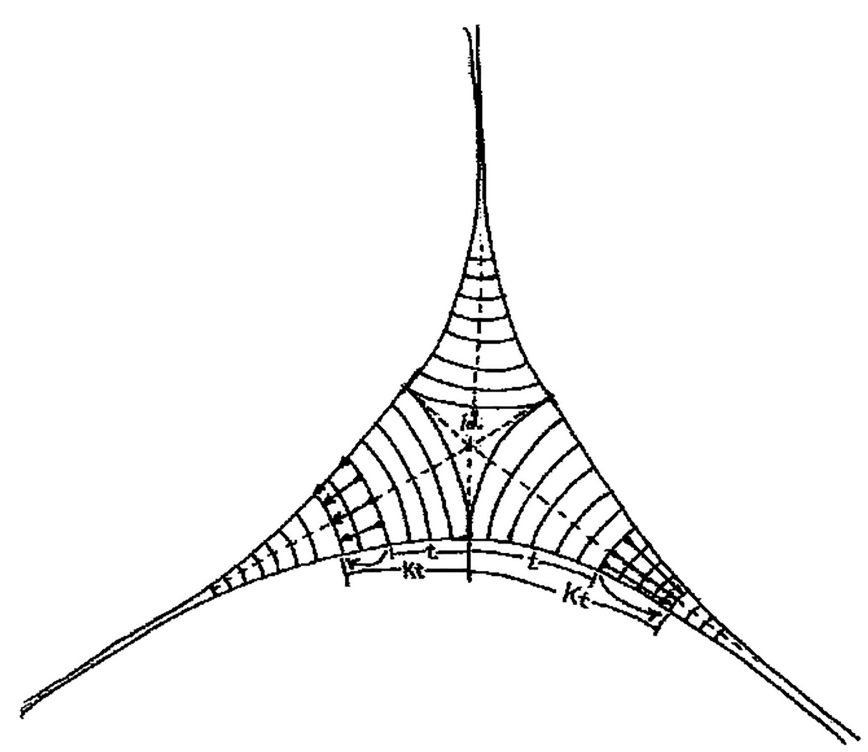

Figure 9. A Lipschitz mapping between ideal triangles; figure extracted from Thurston's paper [19].

Thurston's definition of distortion between surfaces bears some analogy with Lagrange's and Milnor's definitions, but it is global. The definition is as follows: Given a homeomorphism $f$ between two marked hyperbolic surfaces $g$ and $h$, one defines the quantity

$$
L(f)=\sup _{x \neq y} \frac{d_{h}(f(x), f(y))}{d_{g}(x, y)} .
$$

The distance from $g$ to $h$ is set to be

$$
\inf _{f}(\log (L(f))
$$

the infimum being taken over homeomorphisms $f$ between the two surfaces respecting the marking. This distance function satisfies all the axioms of a metric except the symmetry axiom (the distance from $g$ to $h$ is generally different from the distance from $h$ to $g$ ).

Thurston built a comprehensive theory for this distance function, constructing geodesics, showing that this metric is Finsler, describing the infinitesimal norm at each point, etc. He used in an essential way some deformations between hyperbolic surfaces that are built upon simply defined Lipschitz maps between ideal hyperbolic triangles, as shown in Figure 9, where each cuspidal region is foliated by horocycles centered at that cusp. Such a foliation admits a perpendicular foliation by geodesics that converge to the corresponding cusp. The natural coordinates associated with the two-dimensional grid formed by these two orthogonal foliations allows the computation of the Lipschitz constant of the map, indicated in Thurston's drawing: for $K>0$, the map keeps the central unfoliated region untouched and sends a piece of horocycle at distance $t$ from this unfoliated region to a piece of horocycle at distance $K t$. The horocycles are sent linearly onto

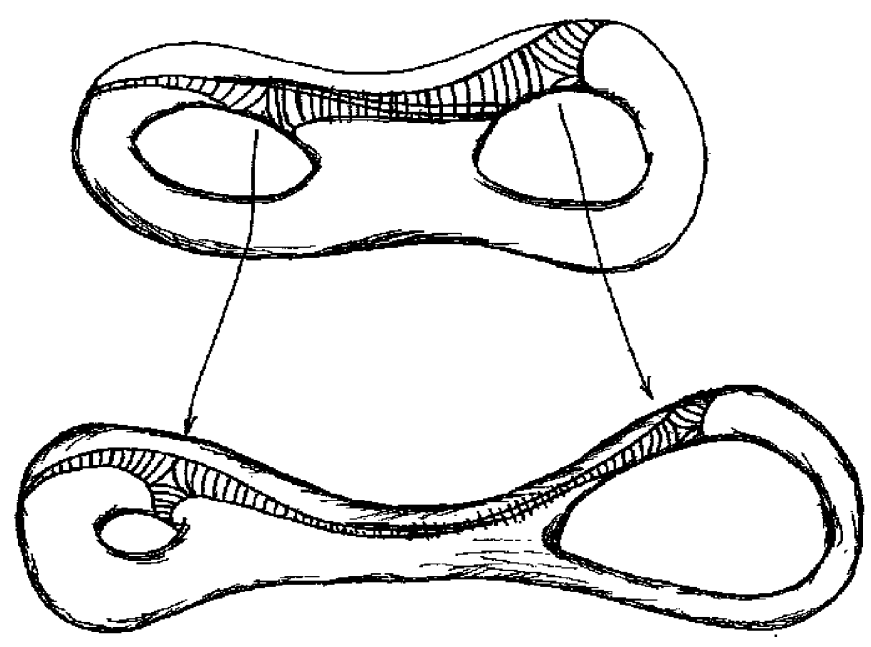

Figure 10. A Lipschitz mapping between two surfaces; figure extracted from Thurston's paper [19].

each other (with respect to their natural parametrization by arclength).

With a proper choice of a decomposition of a hyperbolic surface into ideal triangles, such maps (with the same $K$ ) fit together and define a map from the surface to itself, realizing the best Lipschitz constant between the two hyperbolic metrics (see Figure 10). Varying $K$ gives geodesic lines in Teichmüller space equipped with Thurston's metric.

Thurston's construction may be adapted to the construction of $K$-Lipschitz maps between some hyperbolic right-angled hexagons. Here, the hexagon is equipped with a foliation by hypercycles (lines equidistant to the edges) and by the corresponding foliation by perpendicular geodesics (Figure 11). Again, varying the constant $K$ gives a one-parameter family of geodesic lines in Teichmüller's space; see [15]. The coordinates associated to these orthogonal foliations are used in the computation of the Lipschitz constant of the map and in the proof of the fact that it is optimal. There are very few other cases where best Lipschitz constants of maps between hyperbolic surfaces can be explicitly computed.

In his paper [19], Thurston alludes to the relation between extremal maps between surfaces and cloth deformation. After he states the problem of finding best Lipschitz maps, he writes:

This is closely related to the canonical problem that arises when a person on the standard American diet digs into his or her wardrobe of a few years earlier. The difference is that in the wardrobe problem, one does not really care to know the value of the best Lipschitz constant-one is mainly concerned that the Lipschitz constant not be significantly greater than 1 . We shall see that, just as cloth which is stretched tight develops stress 


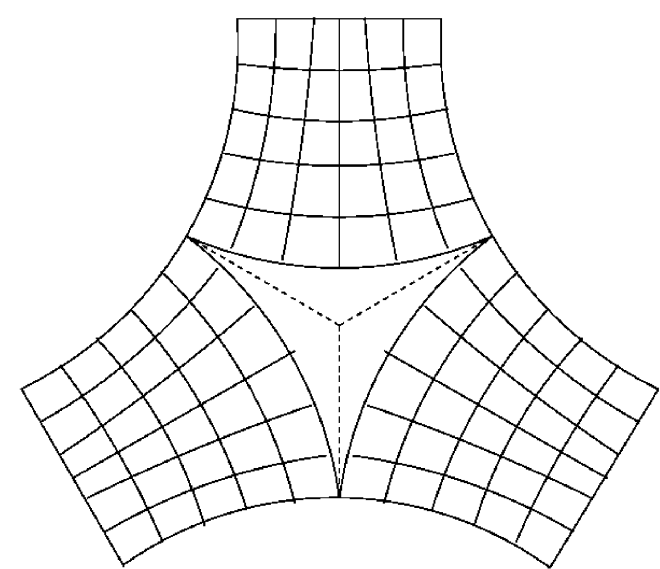

Figure 11. A foliation of the right-angled hyperbolic hexagon by pieces of hypercycles.

wrinkles, the least Lipschitz constant for a homeomorphism between two surfaces is dictated by a certain geodesic lamination which is maximally stretched....

Thurston elaborated on the analogy between extremal maps between surfaces and deformations of garments in several of his lectures.

Talking about garments, we return to Chebyshev, who studied in a mathematical setting the problem of binding a general surface with a piece of fabric. Such a fabric is made out of two perpendicular families of threads, forming a Euclidean net of rectangles. It is designed to cover a surface of a body, a piece of furniture, etc., and optimally-for some kinds of garments: socks, pantyhose, etc.-it should take the form of the surface that it covers. To achieve this, the small rectangles formed by the net are deformed. During this deformation, the lengths of the sides of the rectangles remain constant, but the angles may change. Thus, the rectangles become curvilinear parallelograms.

The problem of drawing geographical maps and that of fitting of garments are in some sense inverse of each other: on the one hand, one searches for best mappings from a curved surface onto a Euclidean one, and on the other hand, one searches for a map from a Euclidean surface onto a curved one.

In the introduction to his first paper on cartography, Chebyshev writes that from the mathematical point of view, the question of finding best geographical maps bears some strong analogy with some problems he considered before concerning linkages. He calls the study of linkages the theory of mechanisms, known under the name of parallelograms. The word "parallelogram" refers here to four-bar linkages that have the form of parallelograms that appear in machines that fascinated Chebyshev, but it also refers to the parallelograms that we mentioned, made by the transverse threads of a garment; the two theories are related. In his second paper on cartography, Chebyshev returns again to the analogy between the theory of parallelograms and cartography, and he talks more precisely about Watt's parallelograms. These are mechanical linkages described by James Watt (1736-1819) in 1784, when the latter patented the so-called Watt steam engine, a machine that transforms the back-and-forth motion of a piston into the rotational motion of a wheel. Chebyshev notes that in both theories (geographical maps and linkages), one looks for a function of two variables that realizes a minimum among functions that satisfy a certain partial differential equation. Let us stop for a while on this analogy.

Given an analytic function $f$, one may approximate it, at a given point, by its sequence of first $n$th Taylor series polynomials. In his work on linkages, Chebyshev was led to the question of finding the best approximation of a function $f$ by a polynomial $P$ on a whole interval and not just a point; that is, he wanted the supremum over that interval of the difference $P(x)-f(x)$ to be minimal. In working on this problem, he found a differential equation that must be satisfied by the desired polynomial. This general scheme is the basis of a series of far-reaching results by Chebyshev that apply to a variety of problems he tackled, including that of geographical maps. He placed the two theories (linkages and geographical maps) in the setting of the calculus of variations, a subject which was dear to Euler and Lagrange.

The name "Chebyshev net" was given to a net made out of quadrilaterals of fixed side length. In a paper titled "On the cutting of garments," ${ }^{17}$ Chebyshev included the problem of the fitting of garments in the setting of the differential geometry of surfaces. The mathematical problem concerns the possibility of covering a surface with a Chebyshev net. The local problem was solved affirmatively by Bieberbach in 1926. It is known that for the global problem, one needs to deal with singularities. Burago, Ivanov, and Malev obtained a general result on the existence of a covering by a Chebyshev net for complete simply connected Alexandrov surfaces under some constraints on the curvature. $^{18}$

Darboux, in his ICM talk in Rome (1908) [4], in which he mentions questions related to geographical maps, also talks about the work of Chebyshev on the fitting of garments. He addresses the following question, which is in the lineage of the questions studied by the Russian geometer: Consider a piece of fabric, in the form of a net that women use to cover their hair. The net is formed by two

\footnotetext{
${ }^{17}$ P. L. Chebyshev, "Sur la coupe des vêtements," Assoc. Franç. pour l'Avancement des Sciences, 7ème session à Paris, 28 Août 1878, 154-155. Reprinted in: P. L. Tchebycheff, Euvres, Vol. 2, p. 708 (excerpt). Reprint, Chelsea, NY. The complete text in Russian appears in the Russian Collected Works.

${ }^{18}$ Y. D. Burago, S. V. Ivanov, and S. G. Malev, "Remarks on Chebyshev coordinates," Zap. Nauchn. Sem. S.-Peterburg. Otdel. Mat. Inst. Steklov. (POMI), 329 (Geom. i Topol. 9) 195 (2005), 5-13.
} 
perpendicular families of threads. These threads are attached at their intersections in such a way that they form small rectangles. The net can be deformed in such a way that the angles at the vertices of the rectangles may vary, but not the side lengths. The question is to determine the form of the net when it is placed on a surface (part of a human body).

Thurston, by the end of his short life, applied his mathematics to the art of haute couture. Around the year 2010, he started working with the Japanese fashion label Issey Miyake on the conception of geometrically inspired garments and more especially on patterns based on nonEuclidean geometry. In an interview (ABC News report) available on the Internet titled "Fashion and advanced mathematics meet at Miyake," along with the designer Dai Fujiwara, Thurston says, "I have long been fascinated (from a distance) by the art of clothing design and its connections to mathematics." In 2010, Issey Miyake presented in Paris a fashion collection with the title "8 Geometry Link Models as Metaphor of the Universe," inspired by Thurston's work. The collection is also known under the name "Poincaré Odyssey." The following is an excerpt of a text of Thurston that was available at the fashion show:

Many people think of mathematics as austere and self-contained. To the contrary, mathematics is a very rich and very human subject, an art that enables us to see and understand deep interconnections in the world. The best mathematics uses the whole mind, embraces human sensibility, and is not at all limited to the small portion of our brains that calculates and manipulates with symbols. Through pursuing beauty we find truth, and where we find truth, we discover incredible beauty.

Talking about Thurston, we also mention the theory of circle packings. He was the main promoter of their use in conformal geometry. In particular, he made a conjecture (later proved by Sullivan and Rodin) on the approximation of the Riemann mapping using circle packings. After this work, deformations of circle packings were used in the algorithmic theory of discrete quasiconformal mappings between surfaces converging to continuous ones. We have chosen Figure 12, extracted from the paper [20], to show an example where circle packings and their deformation play the role of the more familiar Euclidean grid to describe mappings between surfaces.

\section{Brain Warping}

Thurston also realized that conformal mappings may be used in the study of the brain. In a memorial article on him published in the Notices, Ian Agol recounts: "At one point he read a book about vision and the brain, and explained to us how the image from the eye projected onto the brain is approximately a conformal mapping" [7,
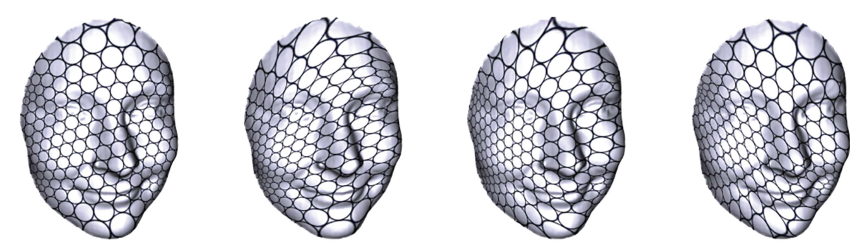

Figure 12. Circle packings used instead of rectangular coordinates as maps between surfaces in the computation of discrete quasiconformal mappings; figure from [20].

\section{p. 36].}

The brain is a singular part of our body where mental images and abstract ideas are created. It is also the center of the connection between our mental and material worlds. It is not surprising that Thurston was highly interested in its functioning.

The brain makes altered images of the reality. This is also a setting where the notion of map distortion may be used. Distortion in the study of the brain occurs at the level of perception as well as at that of brain image registration. Investigations in this field carry the generic name of brain warping. Here, the mathematical study of distortion of maps between surfaces is intertwined with biological research on growth and medical research on pathologies such as Alzheimer's and other neurodegenerative diseases caused by cognitive dysfunction. Aspects in this field include the problem of localizing visual perception processes in specific brain regions and that of transferring images from the brain to a computer, obtaining digital brain images that have medical significance.

A large literature was published in the last few decades on the study of brain alteration and comparison of form, some of it written by mathematicians who used the tools we have on maps between surfaces and their distortion.

As in geography, several kinds of surface transformations are considered in brain warping: angle-preserving, area-preserving, minimal distance-distortion mappings, etc. Classical mathematical techniques have been used by neuro-scientists for computational solutions of such problems, including PDEs, the approximation of Lipschitz mappings by $p$-harmonic maps, and discretization. In several recent papers published on this subject, questions related to brain imaging have been reduced to questions on minimizing quasiconformal and Lipschitz distortions of mappings between surfaces, with boundary conditions specified. In some of these approaches, the surfaces involved represent cortical surfaces, in particular the surfaces between white matter and gray matter or between gray matter and the cerebrospinal fluid in the brain. The problems studied also include the ones of representing on a flat plane and with least distortion the cerebral cortex surface.

There is a rich literature on the subject, and the interested reader may look at the collective book edited by Toga [18] and the paper [13] by Memoli, Sapiro, and Thomp- 
son. Both contain several references.

Back in the fifteenth century, Leonardo da Vinci, defying a papal ban, spent a lot of time dissecting human bodies and made several drawings of the brain. Some of the techniques he introduced were used in three-dimensional digital images; see e.g. [18, p. 299].

I would like to conclude this paper with a quote from Chebyshev $^{19}$ :

Mathematics already traversed two epochs: one where the problems were set by the gods (for instance, the problem of the duplication of the cube) and another one where the semi-gods, like Fermat, Pascal and others, set them. Today we have entered the third period, where the questions to be solved are raised by the needs of humanity.

\section{References}

[1] Beltrami E. Risoluzione del problema: Riportare i punti di una superficie sopra un piano in modo che le linee geodetiche vengano rappresentate da linee rette. Annali di Matematica Pura ed Applicata, 7 (1865), 185-204.

[2] Charitos Ch, Papadoperakis I. On the nonexistence of a perfect map from the 2-sphere to the Euclidean plane, In: Eighteen Essays in Non-Euclidean Geometry, European Mathematical Society, Zurich, 2019, 125-134. MR3965120

[3] Chebyshev PL. Sur la construction des cartes géographiques. Reprinted in P. L. Tchebycheff, Euvres, St. Petersburg, 1899, Vol. 1, 233-236 and 239-247. MR0147353

[4] Darboux G. Les origines, les méthodes et les problèmes de la géométrie infinitésimale. Proceedings of the IVth ICM, Rome, April 6-11, 1908, Tipografia della R. Accademia dei Lincei, Vol. 1, 105-122 (1909).

[5] Euler L. De repraesentatione superficiei sphaericae super plano. Acta Academiae Scientarum Imperialis Petropolitinae, Pars 1 (1777), 107-132. In Opera Omnia: Series 1, Vol. 28, 248-275.

[6] Euler L. De proiectione geographica Deslisliana in mappa generali imperii russici usitata. Acta Academiae Scientarum Imperialis Petropolitinae, Pars 1 (1777), 143-153. Opera Omnia: Series 1, Vol. 28, 288-297.

[7] Gabai D, Kerckhoff S. (eds.), William P. Thurston, 19462012, Notices of the AMS, Part I, Vol. 62, December 2015, No. 11, 1318-1332; Part II, Vol. 63, No. 1, January 2016, 31-41. MR3443712, MR3410780

[8] Gauss CF. Allgemeine Auflösung der Aufgabe: die Theile einer gegebnen Fläche auf einer andern gegebnen Fläche so abzubilden, daß die Abbildung dem Abgebildeten in den kleinsten Theilen ähnlich wird, 1825. In Werke, vol. IV, 189-216.

[9] Grötzsch H. Über die Verzerrung bei nichtkonformen schlichten Abbildungen mehrfach zusammenhängender schlichter Bereiche, Ber. Verhandl. Sächs. Akad. Wiss. Leipzig Math.-Phys. Kl. 82 (1930), 69-80.

\footnotetext{
${ }^{19}$ A. Vassilief, P. Tchébychef et son œuvre scientifique, Boll. di bibliografia e storia delle scienze matematiche, I Turin, 1898, 45-46.
}

[10] Hapgood CH. Maps of the Ancient Sea Kings: Evidence of Advanced Civilization in the Ice Age, Chilton Books, 1966. Paperback edition, Adventures Unlimited Press, 1997.

[11] Lagrange J-L. Sur la construction des cartes géographiques, In Oeuvres de Lagrange, Vol. 4, 637-664 and 664-692.

[12] Lambert JH. Beiträge zum Gebrauche der Mathematik und deren Anwendung, 4 vols., im Verlage des Buchladens der Realschule, Berlin, 1765-1772.

[13] Mémoli F, Sapiro G, Thompson P. Geometric Surface and Brain Warping via Geodesic Minimizing Lipschitz Extensions, 1st MICCAI Workshop on Mathematical Foundations of Computational Anatomy: Geometrical, Statistical and Registration Methods for Modeling Biological Shape Variability, Copenhagen, 58-67, 2006.

[14] Milnor J. A problem in cartography, The American Mathematical Monthly, Vol. 76, No. 10 (Dec. 1969), 1101-1112. MR254791

[15] Papadopoulos A, Théret G. Some Lipschitz maps between hyperbolic surfaces with applications to Teichmüller theory, Geometriae Dedicata, 150, 1 (2011), 233247. MR2994031

[16] Thompson D. On Growth and Form, Cambridge University Press, 1st edition, 1917. MR0006348

[17] Tissot N-A. Mémoire sur la représentation des surfaces et les projections des cartes géographiques, Gauthier-Villars, Paris, 1881.

[18] Toga AW. Brain Warping, Academic Press, New York, 1998.

[19] Thurston WP. Minimal stretch maps between hyperbolic surfaces, preprint (1985) available at arxiv . org/abs/math.GT/9801039.

[20] Zeng W, Lui LM, Luo F, Liu JS, Chan TF, Yau ST, Gu XF. Computing quasiconformal maps on Riemann surfaces using discrete curvature flow, Numer. Math. 121 (2012), no. 4, 671-703. MR2945612

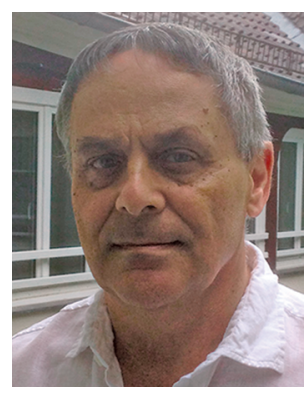

Athanase

Papadopoulos

Credits

Figure 1 is courtesy of Francesco di Antonio del Chierico [Public domain], via Wikimedia Commons.

Figures 2, 4-7, and 11 are courtesy of Athanase Papadopoulos. Figure 3: [Public domain], via Wikimedia Commons.

Figures 8-10 are used with permission.

Figure 12 is courtesy of David Gu.

Author photo is courtesy of the author. 